\title{
Support Vector Regression Methods for Functional Data
}

\author{
Noslen Hernández ${ }^{1}$, Rolando J. Biscay ${ }^{2}$, and Isneri Talavera ${ }^{1}$ \\ 1 Advanced Technologies Applications Center \\ nhernandez@cenatav.co.cu \\ ${ }^{2}$ Institute of Mathematics, Physics and Computation
}

\begin{abstract}
Many regression tasks in practice dispose in low gear instance of digitized functions as predictor variables. This has motivated the development of regression methods for functional data. In particular, Naradaya-Watson Kernel (NWK) and Radial Basis Function (RBF) estimators have been recently extended to functional nonparametric regression models. However, these methods do not allow for dimensionality reduction. For this purpose, we introduce Support Vector Regression (SVR) methods for functional data. These are formulated in the framework of approximation in reproducing kernel Hilbert spaces. On this general basis, some of its properties are investigated, emphasizing the construction of nonnegative definite kernels on functional spaces. Furthermore, the performance of SVR for functional variables is shown on a real world benchmark spectrometric data set, as well as comparisons with NWK and RBF methods. Good predictions were obtained by these three approaches, but SVR achieved in addition about $20 \%$ reduction of dimensionality.
\end{abstract}

Keywords: Support Vector Regression, Functional Data, Kernel Function.

\section{Introduction}

The fast development of instrumental analysis equipment, such as spectrophotometers, chromatographs, signal analyzers and other modern measurement devises, provides huge amount of data as high-resolution digitized functions. As a consequence, regression tasks in which the predictor variable is some type of Functional Data (FD), instead of a low-dimensional vector, are quite common. For example, it is very important nowadays the prediction of chemical physical properties of a product from its spectral function [4, [16].

The direct application of classical multivariate regressions methods for this type of data exhibits serious limitations. Indeed, digitized functions (e.g., spectral data) are generally represented by high-dimensional vectors whose coordinates are strongly correlated. Furthermore, usually the dimension of such vectors greatly exceeds the number of independent observations (e.g., the number of measured spectra). In such situations, standard regression analysis leads to ill-posed inverse problems, which cause a number of difficulties. 
As an alternative, Functional Data Analysis is an extension of traditional multivariate analysis that is specifically oriented to deal with observations of functional nature [12. For this, each individual is characterized by one or more continuous real-valued functions, rather than by a finite-dimensional vector. This allows for applying functional processing techniques such as derivation, integration, etc. On this basis, over the last years several classical multivariate statistical methods have been extended to FD. In respect to regression, first works in this direction were focused on linear models. In particular, least squares methods have been elaborated for linear regression with functional predictors (see, e.g., [1], [12, and references therein). Also, some dimensionality reduction approaches for linear regression, such as principal component regression and partial least squares, have been generalized to FD [1], 3], 9]. More recently, a number of estimation methods for functional nonparametric regression models have been also introduced. Namely, estimators based on functional data adaptations of classical neural networks 13, Naradaya-Watson Kernel (NWK) estimators 6], [7] and regularization in Reproducing Kernel Hilbert Spaces (RKHS) 9] - the latter including Radial Basis Function (RBF) methods. Likewise their classical counterparts in multivariate analysis, these nonparametric techniques provides high flexibility to approximate a wide class of functional regressions. However, a difficult with them is that a high amount of computer memory is required to encode the estimates in order to make future predictions (e.g., the whole data set has to be retained for predicting by NWK and RBF methods).

In the present paper, the drawback just mentioned is approached by introducing Support Vector (SV) regression methods on functional spaces. Though SV regression have been studied by several authors in the more general framework of abstract RKHS methods (see, e.g., [15], [5], 2]), to our knowledge no work have been carried out to deal with its application to FD. On the basis of the RKHS framework we study some properties of SV regression for FD, emphasizing specificities related with the construction of nonnegative definite (nnd) kernels on functional spaces. Furthermore, the feasibility and practical performance of $\mathrm{SV}$ regression for FD, as well as comparisons with NWK and RBF estimators, are shown on a real world benchmark spectrometric data set.

\section{Estimation of Abstract Nonparametric Regression Models by Regularization in Reproducing Kernel Hilbert Spaces}

Estimation methods for very general regression models have been elaborated by several authors on the basis of regularization in RKHS (see, e.g., [15, 5], 2], and references therein, for definitions and detailed expositions). We will here briefly describe this abstract framework before considering its specification for regression with FD in the next Section.

Let $\mathcal{X}$ be a linear space with norm \|\|$_{\mathcal{X}}$, and $\mathbb{R}^{\mathcal{X}}$ be the set of functions from $\mathcal{X}$ into $\mathbb{R}$. Suppose it is given some positive definite (pd) function (or kernel) $\kappa: \mathcal{X} \times \mathcal{X} \rightarrow \mathbb{R}$. It is known that there exists a RKHS $\mathcal{H}_{\kappa} \subset \mathbb{R}^{\mathcal{X}}$ with reproducing 
kernel $\kappa$. The norm on $\mathcal{H}_{\kappa}$ will be denoted by \|\|$_{\mathcal{H}_{\kappa}}$. Consider the abstract nonparametric regression model:

$$
Y=\Psi(X)+e,
$$

where $(X, Y)$ is a random variable on some probability space $(\Omega, \mathcal{F}, P)$ with values in $\mathcal{X} \times \mathcal{Y}, \mathcal{Y} \subset \mathbb{R}, e$ is a real-valued random variable with zero mean, which is assumed to be independent from $(X, Y)$, and $\Psi$ is an unknown mapping $\mathcal{X} \rightarrow \mathbb{R}$. The variables $X$ and $Y$ are interpreted, respectively, as the predictor and response variable in this regression model. The problem of interest is to estimate the regression mapping $\Psi$ on the basis of data $\left(X_{i}, Y_{i}\right), 1 \leq i \leq n$, formed by independent and identically distributed observations of $(X, Y)$.

For this, let $\mathcal{H}_{0}$ be a given finite-dimensional linear subspace of $\mathbb{R}^{\mathcal{X}}$ with basis $G_{1}, \ldots, G_{m}$, such that the matrix $\left(G_{j}\left(X_{i}\right)\right), 1 \leq i \leq n, 1 \leq j \leq m$, has rank $m$. Denote by $\mathcal{H}=\mathcal{H}_{\kappa}+\mathcal{H}_{0}$ the space of functions $F=F_{\kappa}+F_{0}$ with $F_{\kappa} \in \mathcal{H}_{\kappa}$ and $F_{0} \in \mathcal{H}_{0}$. Henceforth, it will be assumed that $\Psi \in \mathcal{H}$. To assess the closeness of each $F \in \mathcal{H}$ to the unknown $\Psi$, suppose that a function $c: \mathcal{X} \times \mathcal{Y} \times \mathcal{H} \rightarrow \mathbb{R}_{+}$ (called a contrast function) has been specified. The risk of approximating $\Psi$ by an element $F=F_{\kappa}+F_{0} \in \mathcal{H}$ is then quantified by

$$
R(F)=E(c(X, Y, F(X))),
$$

where $E$ is the expectation with respect to the distribution of $(X, Y)$. The corresponding empirical risk and the regularized empirical risk are defined, respectively, as

$$
R_{e m p}(F)=\frac{1}{n} \sum_{i=1}^{n} E\left(c\left(X_{i}, Y_{i}, F\left(X_{i}\right)\right)\right)
$$

and

$$
R_{\lambda}(F)=R_{e m p}(F)+\lambda\left\|F_{\kappa}\right\|_{\mathcal{H}_{\kappa}},
$$

where $\lambda \geq 0$ is a a given constant (regularization hyperparameter). The regularized estimate $\widehat{\Psi}_{\lambda}$ of $\Psi$ is defined through the optimization problem

$$
\widehat{\Psi}_{\lambda}=\arg \min _{F \in \mathcal{H}} R_{\lambda}(F) .
$$

As has been pointed out by several authors, this general framework provides an unified treatment of several approaches to nonparametric regression, in dependence of the specification of the contrast function $c$ (see,e.g., 5] and references therein). In particular:

c1) Regularized least squares regression methods correspond to the quadratic contrast

$$
c(X, Y, F(X))=(Y-F(X))^{2} .
$$

More particularly, standard $\mathrm{RBF}$ regression methods are obtained when the quadratic contrast is adopted, $\mathcal{X}$ is a subspace of $\mathbb{R}^{d}$ (for some $d \in \mathbb{N}$ ), and the kernel has the radial form $\kappa(x, x)=g(\|x\|)$ for some function $g: \mathbb{R}_{+} \rightarrow \mathbb{R}$. Here, $\|x\|$ is the euclidean norm on $\mathbb{R}^{d}$. 
c2) Support Vector regression methods correspond to the so-called $\epsilon$-insensitive contrast function

$$
c(X, Y, F(X))=|Y-F(X)|_{\epsilon}=\max (|Y-F(X)|-\epsilon, 0),
$$

where $\epsilon \geq 0$ is some given constant. In this case, $\mathcal{H}_{0}$ is taken as either the onedimensional space generated by the constant function $G_{1}(x) \equiv 1$ or $\mathcal{H}_{0}=\{0\}$.

\section{Support Vector Estimators for Functional Nonparametric Regression}

The general framework for regression estimation through regularization in RKHSs, as sketched above, can be applied for any specification of the space $\mathcal{X}$, the pd kernel $\kappa$ on $\mathcal{X}$, the contrast function $c$, and the finite-dimensional subspace $\mathcal{H}_{0}$ of $\mathbb{R}^{\mathcal{X}}$.

Classical applications of RKHS methods for regression deal with situations in which $\mathcal{X} \subset \mathbb{R}^{d}$, and so $\mathcal{H} \subset \mathbb{R}^{\mathcal{X}}$ is constituted by multivariate functions $F: \mathcal{X} \subset \mathbb{R}^{d} \rightarrow \mathbb{R}$. This is the setting of multivariate regression methods, in which the regression mapping $\Psi$ to be estimated is a multivariate function, i.e., a function on $\mathbb{R}^{d}$.

On the contrary, functional nonparametric regression models deal with cases in which $\mathcal{X} \subset \mathbb{R}^{\mathcal{T}}$ is a set of functions $x: \mathcal{T} \rightarrow \mathbb{R}$, where $\mathcal{T}$ is an infinitedimensional set. For example, in some practical situations of interest, $\mathcal{T}=[a, b]$ is a closed interval in $\mathbb{R}$, and $\mathcal{X}$ is the space $L^{2}([a, b], \mathbb{R})$ of squared-integrable functions defined on $[a, b]$. Thus, in regression models with FD the unknown regression mapping $\Psi$ is a functional defined on a normed space $\mathcal{X}$ of real-valued functions.

The estimation of functional regression models by RKHS methods have been recently initiated in 9] under the quadratic contrast, hence providing regularized least squares estimates. These are instances of the general class $(c 1)$ described in the previous Section.

In the present work we introduce methods to estimate regression models for FD adopting the $\epsilon$-insensitive contrast function, i.e. we adapt to functional data the general approach $(c 2)$ mentioned above.

It is known that under quite general conditions on the contrast function $c$ (which are satisfied by both the quadratic and the $\epsilon$-insensitive contrasts), the regularized estimate $\widehat{\Psi}_{\lambda}$ in any abstract regression model has the following explicit form: for all $x \in \mathcal{X}$,

$$
\widehat{\Psi}_{\lambda}(x)=\sum_{i=1}^{n} a_{i} \kappa\left(x_{i}, x\right)+\sum_{j=1}^{m} b_{j} G_{j}(x)
$$

for some $a_{i}, b_{j} \in \mathbb{R}$ that depend only on the hyperparameter $\lambda$ and the matrices $K=\left(\kappa\left(x_{i}, x_{j}\right)\right), G=\left(G_{j}\left(x_{i}\right)\right)$.

Therefore, once the kernel $\kappa$ is given, the numerical computation of RKHSbased regularized estimators $\widehat{\Psi}_{\lambda}$ in regression for FD input (i.e., when $\mathcal{X}$ is a 
space of functions) is exactly the same as in regression for multivariate input (i.e., when $\mathcal{X}$ is a subspace of some finite-dimensional linear space $\mathbb{R}^{d}$ ).

Furthermore, both for quadratic and $\epsilon$-insensitive contrast functions ( i.e., for regularized least squares and SV regression approaches), many properties of the estimator $\widehat{\Psi}_{\lambda}$ in the FD setting can be quite directly derived from known results in the framework of abstract regression models. For example, if $\mathcal{Y}$ is bounded by a constant $M_{\mathcal{Y}}$ and $\kappa$ is bounded by a constant $M_{\kappa}$ then, for any $\lambda \geq 0$,

$$
R_{e m p}\left(\widehat{\Psi}_{\lambda}(x)\right)-R\left(\widehat{\Psi}_{\lambda}(x)\right) \rightarrow 0
$$

in probability exponentially fast as $n \rightarrow \infty$ (10, see also [2] and 15]). Likewise, some results on uniform convergence in probability

$$
R_{e m p}(F)-R(F) \rightarrow 0
$$

over sets of the form $\left\{F:\|F\|_{\mathcal{H}_{\kappa}} \leq A\right\}, A \in \mathbb{R}$, are discussed e.g. in [5].

However, RKHS-based regularization has important specificities in case of regression models for functional data. Some of them, mainly related with the interpretation and construction of pd kernels on functional spaces, are discussed bellow.

\section{Nonnegative Definite Kernels on Functional Spaces}

Nonnegative definite (nnd) kernels on finite-dimensional linear spaces $\mathbb{R}^{d}$ are usually constructed on the basis of classical differential operators or completely monotone functions of order $q \in \mathbb{N}$, from which standard spline and RBF approximations to multivariate functions are derived [18. Unfortunately, these approaches are not directly applicable to obtain nnd kernels on infinite-dimensional spaces. This difficulty poses a challenge to the RKHS approach to regression for FD.

In this Section we will give some results that allow for the construction of some classes of nnd kernels on functional spaces. It will be assumed that $\mathcal{X}$ is some (infinite-dimensional) separable normed space. Thus, all results are applicable to typical problems with FD, in which $\mathcal{X} \subset \mathbb{R}^{\mathcal{T}}$ and $\mathcal{T}$ is an interval of the real line. Notations and definitions introduced in the previous Sections are assumed.

The following lemma can be easily proved.

Lemma 1. Let

$$
\varphi(x)=E\left(e^{i W(x)}\right)
$$

be the characteristic function of a stochastic process $(W(x): x \in \mathcal{X})$ on $\mathcal{X}$. If $x \rightarrow W(x)$ is a linear functional, and if for all $x \in \mathcal{X}$, the distribution of the

random variable $W(x)$ is symmetric around zero, then

$$
\kappa\left(x, x^{\prime}\right)=\varphi\left(x-x^{\prime}\right)
$$

is a (real-valued) nnd kernel on $\mathcal{X}$. 
A direct consequence of this lemma is the following well-known fact. If $\mathcal{X}$ is a separable Hilbert space and $h \succ 0$ then the Gaussian function

$$
\varphi(x)=e^{-\frac{1}{h}\|x\|^{2}}
$$

defines a nnd kernel on $\mathcal{X}$ (called the Gaussian kernel [15]) by means of

$$
\kappa\left(x, x^{\prime}\right)=\varphi\left(x-x^{\prime}\right)=e^{-\frac{1}{h}\|x-x\|^{2}} .
$$

Indeed, it is known that there exists a probability space $\Omega$ and a Gaussian and linear stochastic process $(W(x): x \in \mathcal{X})$ on $\Omega$ with zero mean (called the isonormal or Gaussian process on $\mathcal{X}$ ) such that

$$
E\left(W(x) W\left(x^{\prime}\right)\right)=\left(x, x^{\prime}\right)
$$

where $(x, x)$ is the scalar product in $\mathcal{X}$. Hence, the conditions of the lemma 1 are satisfied by taking

$$
\varphi(x)=E\left(e^{i W(x)}\right)=e^{-\frac{1}{h}\|x\|^{2}} .
$$

Lemma 1 provides other interesting classes of nnd kernels, as the one stated in the following theorem.

Theorem 1. Let $\mathcal{T}$ be an interval of $\mathbb{R}, \alpha \in(0,2], h \geq 0$, and $\mathcal{X}$ be a set of measurable functions $\mathcal{X} \subset \mathbb{R}^{\mathcal{T}}$ such that

$$
\|x\|_{L_{\alpha}}=\left(\int_{\mathcal{T}}|x(t)|^{\alpha} d t\right)^{\frac{1}{\alpha}}
$$

is finite for each $x \in \mathcal{X}$. Then,

$$
\kappa(x, x)=e^{-\frac{1}{h}\|x-x\|_{L_{\alpha}}^{\alpha}}
$$

is a nnd kernel on $\mathcal{X}$ (which we will call an " $\alpha$-stable kernel").

Indeed, define

$$
W(x)=\frac{1}{h} \int_{\mathcal{T}} x(t) d Z(t),
$$

where $(Z(t): t \in \mathcal{T})$ is a standard symmetric $\alpha$-stable Levy motion 14 Then, it can be shown that $W$ satisfies the conditions of lemma 1 .

Notice that, more generally, also as a consequence of lemma[1] if the stochastic process $(Z(t): t \in \mathcal{T})$ is any semimartingale whose increments have symmetric distributions, and if the stochastic integral (1) is well-defined for all $x \in \mathcal{X}$, then

$$
\kappa\left(x, x^{\prime}\right)=E\left(e^{i(W(x)-W(x))}\right)
$$

is a nnd kernel.

The investigation of the variety of kernels for FD provided by these results is an interesting issue for further research. As a first step in this direction, in the next Section we will restrict to explore the performance of SV regression with Gaussian kernels on a real FD set. 


\section{Application to Real Data}

The main objective in this section is to demonstrate the feasibility of Support Vector Regression (SVR) for FD, and its behavior regarding RBF and NWK estimators.

The considered experimental data comes from the food industry named Tecator [17, It consist of 215 near-infrared absorbance spectra of meat samples, recorded on a Tecator Infratec Food and Feed Analyzer. Each observation consists in a 100-chanel absorbance spectrum in the 850-1050 nm wavelength range. Each spectrum in the data base is associated to a content description of meat sample, obtained by analytic chemistry; the percentage of fat, water and protein are reported. The regression problem consists in the prediction of the fat percentage from the spectrum.

From the 215 spectra, 43 are kept aside as a testing set and the 172 remaining sample is used for model estimation (training set). It should be mentioned that the spectra are finely sampled, leading to very smooth curves. Each function was represented by a 4 th order B-spline approximation with 32 basis functions. The adopted kernel was the Gaussian kernel with the $L_{2}$ norm of the second derivative of the spectrum. This norm was computed on the basis of the Bspline representation of the spectrum. All calculations were performed using Matlab. For SVR, the LIBSVM implementation embedding in a Matlab toolbox was used.

Table 1. Prediction accuracy for different regression estimators

\begin{tabular}{|c|c|c|c|c|}
\hline & rmse & max Abs Error & max. Rel. Error & Avrg. Rel. Error \\
\hline SVR & 0.5451 & 1.2933 & 0.1653 & 0.0276 \\
\hline RBF & 0.9279 & 2.8486 & 0.3061 & 0.0512 \\
\hline NWK & 1.4563 & 5.6000 & 0.2196 & 0.0642 \\
\hline
\end{tabular}

In order to select best hyperparameter values $(\lambda, \epsilon, h)$ to train the SVR, 10 folds Cross-Validation over a grid search was done. For RBF and NWK estimates, Generalize Cross-Validation [18 was applied to tune the hyperparameters.

Figure 1 contains plots of predicted values versus real values of the response variable in the testing sample for each regression method: SVR, RBF and NWK. It is clear that prediction accuracies (generalization capabilities) are quite good for all these methods but SVR seems to exhibit the best one.

This is quantitatively confirmed in Table1 which summarizes the values of several measures of prediction accuracy over the testing sample for each method: root mean squared error (rmse), maximum absolute error (max Abs Error), maximum relative error (max. Rel. Error) and average relative error (Avrg. Rel Error). SVR shows the best predictive behavior in terms of all error measures while the NWK method is the worst one, particularly in respect to the maximum absolute error. 


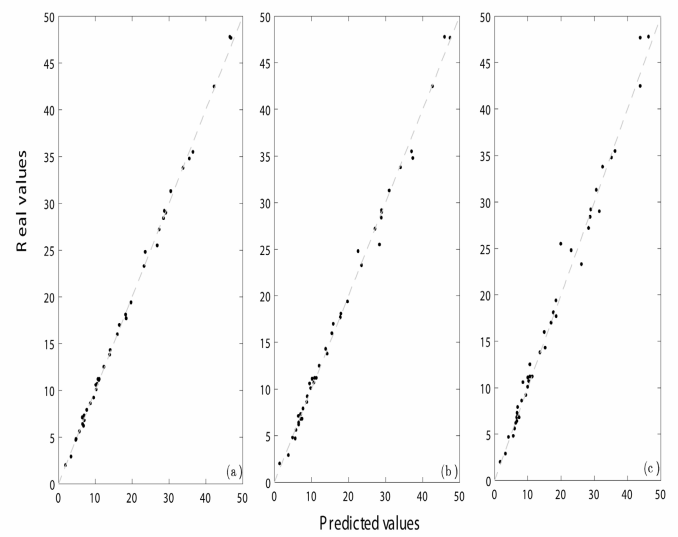

Fig. 1. Real values vs. Predicted values for a) SVR, b) RBF, and c) NWK estimates

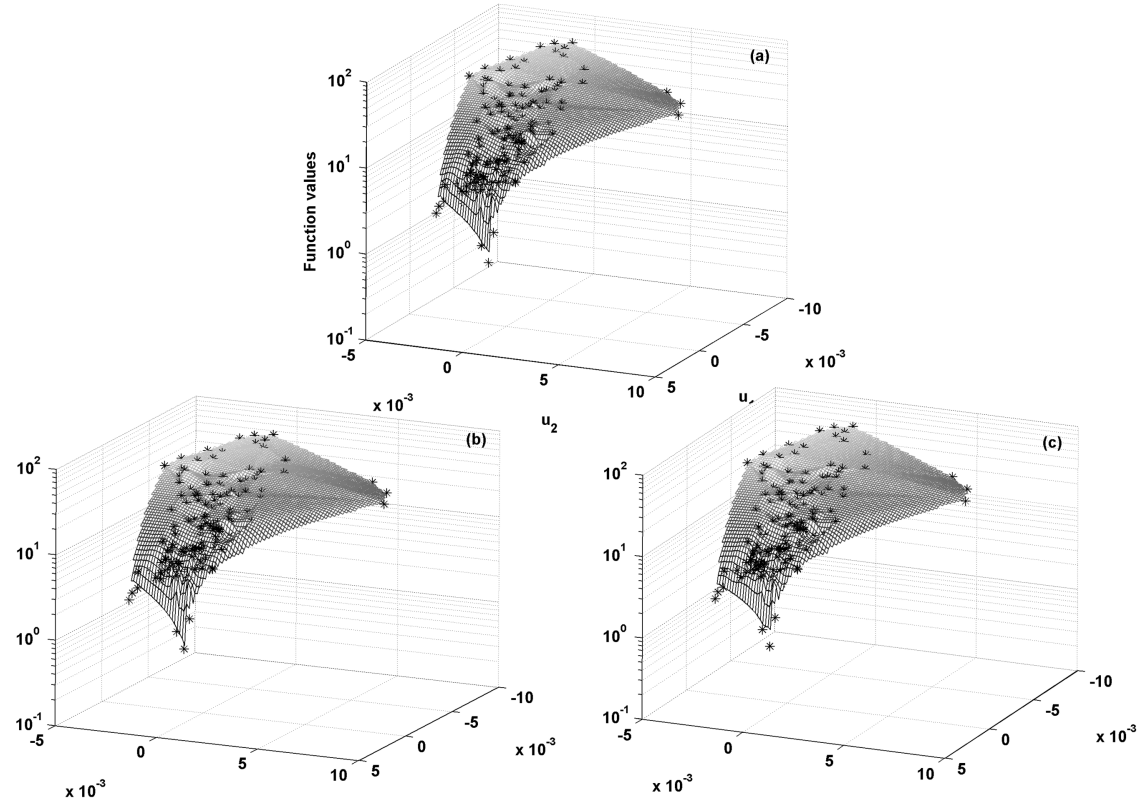

Fig. 2. Functional regression estimates for (a) SVR, (b) RBF, (c) NWK estimator

In order to aid to a better understanding of the different regression estimators in terms of prediction capabilities and shapes of the regression estimates, a graphic representation of the fitted regression functional would be convenient. However, their complete visualization is not possible due to the fact that they live in an infinite dimensional space. To overcome this, a (metric) multidimensional scaling transformation to the two-dimensional euclidean space was applied 
to the inter-distance matrix among all spectra (distances computed according to the $L_{2}$ norm of second derivatives mentioned above). In this way each spectra $x_{i}$ is (approximately) represented by a point $\left(u_{i}, v_{i}\right)$ in the plane. For each method, Figure 2 shows the (interpolated) surface formed by the predicted values $\widehat{y}_{i}$ (vertical axis) as a function of the multidimensional scaling representations $\left(u_{i}, v_{i}\right)$, $i=1, \ldots, n$ (in the horizontal plane). The points $\left(u_{i}, v_{i}, y_{i}\right)$ that represents the original fat percentage values $y_{i}$ corresponding to the spectra $x_{i}$ are also plotted as asterisks.

It is observed that Figure 2 further corroborates the nice goodness of fit attained by the three estimation methods considered, as well as the shape similarity of their estimates of the regression functional.

However, two advantages of SVR should be highlighted. First, it showed a slightly better prediction accuracy as reflected by all error measures in Table 1 . Second, SVR achieved about $20 \%$ of dimensionality reduction by retaining from the original data $(100 \%)$ only a proper subset of selected support vectors (80 $\%$ ). This is in contrast to the other two methods that require to keep all the training data to predict a future sample.

\section{Conclusions and Final Remarks}

This paper demonstrates the theoretic and practical viability of SVR methods for functional data inputs.

A comparison of the prediction errors among SVR and two other regression methods for functional nonparametric regression, namely RBF and NWK estimators, is presented on a spectroscopy benchmark data set. SVR exhibits the best performance in terms of absolute and relative measures of prediction accuracy, closely followed by RBF. An additional advantage of SVR was its capability for dimensionality reduction, which was around $20 \%$ in the analyzed data set. This in contrast to the other two methods that must retain all the training data in order to make a prediction for a new sample.

Notwithstanding, it should be noted that computing SVR estimates involves slightly larger learning times and number of tuning hyperparameters than RBF and NWK methods.

On the theoretic side, we emphasize the important open problem of developing methods for constructing nnd kernels with desired smoothness properties on infinite-dimensional functional spaces. Results derived in the present paper offer some classes of nnd kernels for functional data, such as $\alpha$-stable kernels that have long tails. Further research is necessary to explore their practical relevance, and to widen the toolkit of nnd kernels for FD.

We also recommend to take into account in future investigations on similar data sets the possible influence of spectral preprocessing in the quality of regression estimates.

We conclude that SVR for FD appears as a promising and competitive tool for functional nonparametric regression tasks in practice due to its approximation flexibility and its capability for dimensionality reduction. 


\section{References}

1. Aguilera, A.M., Ocana, F., Valduama, M.J.: An approximated principal component prediction model for continuous-time stochastic processes. Appl. Stochastic Models Data Anal. 13, 61-72 (1999)

2. Bousquet, O., Elisseef, A.: Stability and Generalization. J. of Machine Learning Research 1 (2002)

3. Cardot, H., Ferraty, F., Sarda, P.: Functional linear model Statist. Probab. Lett. 45, 11-22 (1999)

4. Geladi, P.: Chemometrics in spectroscopy: Part 1. Classical Chemometrics. Spectrochem. Acta, Part B 58, 767-782 (2003)

5. Eugeniou, T., Pontil, M., Poggio, T.: Regularization networks and Support Vector Machines. In: Smola, A.J., Bartlettt, P.L., Schölkopf, B. (eds.) Advances in Large Margin Classifiers, pp. 171-203. The MIT Press, Cambridge (2000)

6. Ferraty, F., Vieu, P.: Nonparametric models for functional data with application in regression, time series prediction and curve discrimination. J. Nonparametric Statist. 16, 11-125 (2004)

7. Ferraty, F., Vieu, P.: Nonparametric Functional Data Analysis. Springer Series in Statistics (2006)

8. Frank, J., Friedman, J.H.: A statistical view of some chemometrics regression tools. Technometrics 35, 109-148 (1993)

9. Preda, C., Saporta, G.: PLS regression on stochastic processes. Comput. Statist. Data Anal. 48, 149-158 (2005)

10. Preda, C.: Regression models for functional data by reproducing kernel Hilbert space methods. J. Statistical Planning and Inference 137, 829-840 (2007)

11. Some tools for functional data analysis. J. R. Stat. Soc. Ser. B 53, 539-572 (1991)

12. Ramsay, I., Silverman, B.: Functional Data Analysis. Springer, Heidelberg (1997)

13. Rossi, F., Conan-Gues, B.: Functional Multilayer perceptron: a nonlinear tool for functional data analysis. Neural Networks 18, 45-60 (2005)

14. Samorodnitsky, G., Taqqu, M.S.: Stable Non-Gaussian Random Processes. Chapman \& Hall, New York (1994)

15. Schölkopf, B., Smola, A.: Learning with Kernels. The MIT Press, Cambridge (2002)

16. Swierenga, H.: Multivariate Calibration Model in Vibrational Spectroscopic Applications. PhD. Thesis, Univ. of Nijmegen, Nijmegen (2000)

17. Tecator dataset. available on statlib: http://lib.stat.cmu.edu/datasets/ tecator

18. Wahba, G.: Spline Models for Observational Data. SIAM, Philadelphia (1990) 